Asian Journal of Information Technology 11 (2): 71-76, 2012

ISSN: $1682-3915$

(C) Medwell Journals, 2012

\title{
A Future Ready Smart Network
}

\author{
Debajyoti Pal \\ Camellia Institute of Technology, 700129 Kolkata, India
}

\begin{abstract}
The complexity of home networks has evolved to a greater level of sophistication and complicacy in the recent times comprising of heterogeneous components like at least two computers, web-enabled high-definition television sets, net-enabled blue ray disc players, iPods and many other such devices. Troubleshooting such a sophisticated smart home network in case of a malfunction by the novice end users seems to be very demanding. The study proposes a Smart Home Network Monitoring System that provides a centralized, general-purpose, automatic and convergent logging facility with the purpose to auto-detect and possibly correct all such failure issues by having a well-defined set of adaptive and incremental rule engine that needs to be applied to the entire network in general. Logging of all events that happened before trouble appeared may give a greater insight and hence help in providing an effective and permanent troubleshooting mechanism. This study also reports the initial experience of deploying such a facility.
\end{abstract}

Key words:Future Home Network System, general-purpose logging facility, adaptive and incremental rule-engine, event, troubleshooting

\section{INTRODUCTION}

Penetration of cheap broadband service in the past few years has lead to a surge in the home networking environment and subsequently the problems associated with it are becoming well-known. The ultimate goal of providing an integrated multimedia entertainment service has resulted in the emergence of smart home networks consisting of a number of sophisticated yet complex products like laptops, HDTV, Blue-ray disc players and tablets, etc. that have ultimately created a plethora of problems for the ultimate home users. In fact the problems that plague the smart home networks though simple are a cause of great confusion and frustration among the end users because of their lack of knowledge and expertise (Grinter et al., 2005; Sheehan et al., 2008; Chetty et al., 2007). Misconfigured home networks are a great deal of concern from the security point of view also because they serve as attractive trap-doors for external attackers to exploit. The causes for home network failure can be many and thus tools and logging facilities that enable us to automatically monitor, record, detect and correct such issues will be welcomed.

Continuous monitoring and logging of home network traffic (both inward and outward) can be helpful to provide an insight to the problems that arise in such a network. Specifically what event (s) led to the malfunctioning of the home network might come into limelight by maintaining such a log and can come to be handy in designing an automated, adaptive and incremental self-diagnostic rule matching system (engine).
Packet-monitoring tools like tcpdump, Wireshark, Kismet, etc. helps us to monitor and log all the incoming and outgoing network traffic. All of them however suffer from the same drawback of being tied down to one specific host at a time. Further in most of the homes presence of a NAT enabled router/gateway for establishing an Internet connection to the ISP server complicates the issue in the sense that it renders the outward traffic monitoring useless. Also due to being tied down to one specific host, multiple tools need to be present one for each host that is a part of the smart home network. This clearly gives rise to redundant data that serves as a bottleneck for the bandwidth which is shared between the different active devices.

In this study, researchers propose a centralized, general-purpose, automatic and convergent logging facility that serves as a basis to auto-detect and correct all possible smart home network failures. Since, researchers used Wireshark as the packet monitoring tool hence a centralized logging facility is required so as to ensure that redundant data flow and hence bandwidth clogging is minimized. Thus, the home network implies the presence of client/server architecture which ensures that all the incoming/outgoing traffic is forced to pass through the centralized device that houses the packet monitoring tool. The logging platform is a general purpose one because not only does the packet monitoring tool researchers deploy operating system neutral but it also supports a wide variety of network protocols from the application, transport, network and data-link layers and of the TCP/IP stack. The logging facility is automatic because the packet 
monitoring tool at all times is running in the background and storing the events in a specified location of the storage disk. When a particular limit of the disc usage space is reached the recorded events are transferred to another secondary storage medium. The overall reliability of the system is increased by having the idea of primary/secondary storage in the event of primary storage failure. The centralized architecture that researchers follow automatically forces the entire system to be a convergent one because traffic from all possible locations are ultimately redirected to a centralized server that researchers already discussed. The aforesaid facility has got a close resemblance with a typical Black-box present in the aircrafts and researchers refer to the system that houses the monitoring, logging and troubleshooting facility as the Future Home Network System (FHNS).

\section{FHNS SYSTEM CONFIGURATION AND FUNCTIONALITY}

The place of deployment of the FHNS logging facility is of utmost importance. Researchers follow the typical Client/Server Architecture Model (Calvert et al., 2007) wherein a single system houses the FHNS facility and all the outgoing or incoming network traffic of any kind must flow through it. Such a scheme has been shown in Fig. 1. The configuration has provision both for wired as well as wireless devices. Since, the total number of active network points can go well beyond 5 very easily we choose a 10 port Ethernet switch for the wired section which in turn is connected to one of the Ethernet ports of the FHNS System. Wireless support is also provided by the FHNS System directly in the form of IEEE $802.11 \mathrm{a} / \mathrm{b} / \mathrm{g} / \mathrm{n}$ standards. Although, home networks with a more complex configuration can do exist but researchers assume to be a sufficient one for at-least a couple of years to come by. Figure 2 shows the overall FHNS system functionality. As shown the FHNS System can be subdivided into 4 blocks namely:

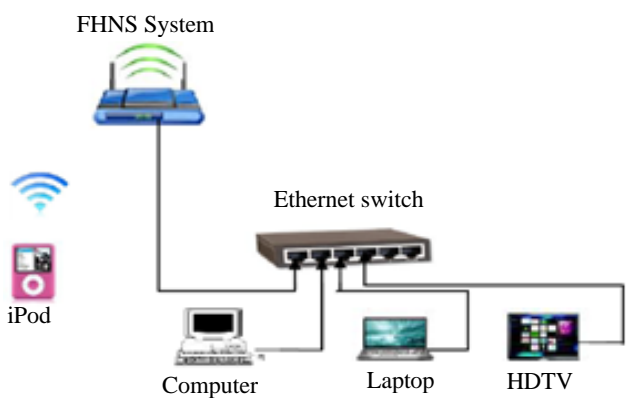

Fig. 1: FHNS System configuration and functionality scheme

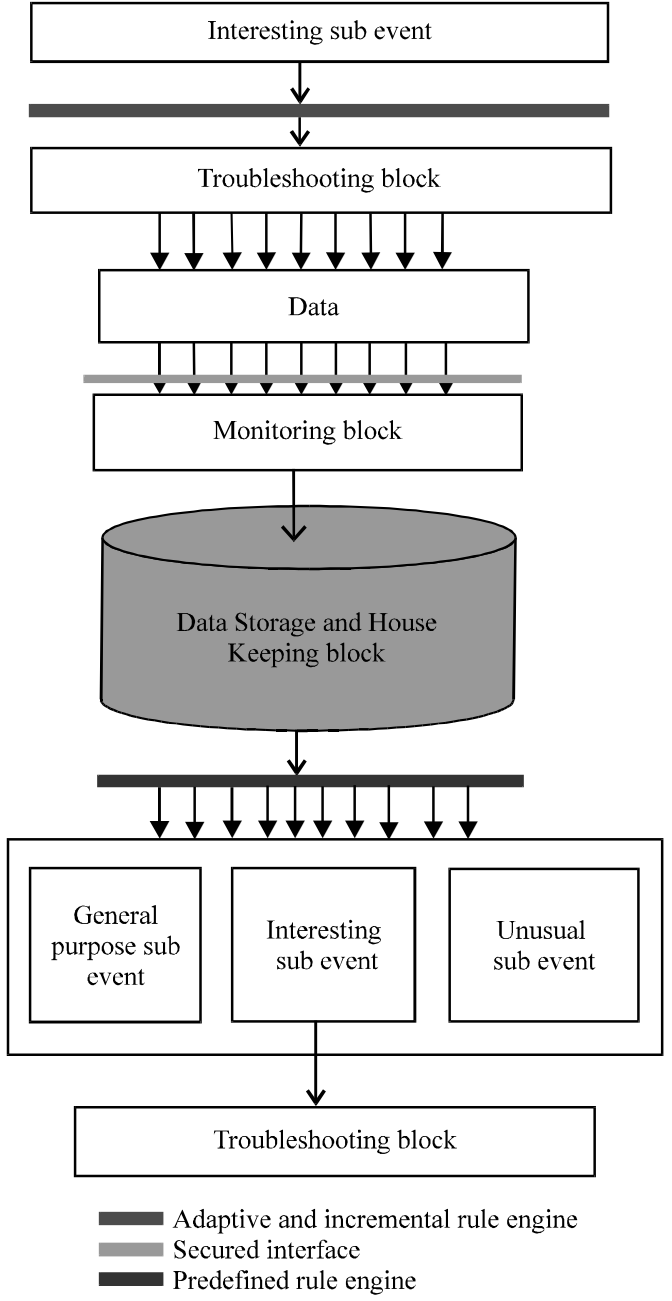

Fig. 2: The overall FHNS System functionality

Monitoring block: It actually houses the packet monitoring software like Wireshark which is responsible for capturing all the home network data that are being generated and subsequently transmitted.

Data Storage and Housekeeping block: This block is responsible for storing all the packets that are being sensed by the Monitoring block. Each and every packet is opened up and depending upon its contents a predefined rule-set is applied and the packets are transferred to a proper Event Generation block.

Event Generation block: It actually can be subdivided into the following sub-blocks:

- General-Purpose Event Sub-block which contain the logs of all the incoming and outgoing network traffic under normal and healthy network operating conditions (no network malfunction) 
- Unusual Event Sub-block which contains the logs of some rare network traffic like a new MAC address appearing for the first time or modification of the configuration settings of a file that is rarely touched

- Interesting Event Sub-block which contains the logs of certain filtered network traffic that might be attempting to update an operating system, updating some antivirus software or searching for device driver software's for a newly installed piece of hardware (maybe like a graphics card) or any other such related items

- Troubleshooting Action block is a block that has access to the Interesting Event Sub-block and is of prime importance. It houses specialized application program that takes appropriate troubleshooting measures if such a condition is detected. Thus, this sub-block should obviously have access to all the sensitive user-data also that might pose to be a security threat or a breach of privacy. Hence, the interface that is used by this sub-block to access the user-data should be done through a secure channel as depicted by a dotted line in Fig. 2

The primary application of the FHNS System is to provide support for troubleshooting and diagnosis when some things fail on a smart home network. If the FHNS System is widely adopted then such a service might be provided by a third party provider or by the ISP itself as a value added service on a chargeable basis.

\section{APPLICATIONS OF FHNS SYSTEM}

In this study researchers consider some applications of the FHNS System.

Automatic troubleshooting and future prediction: This obviously is the prime reason to have the FHNS System in place. Studies by Sheehan shows that end-users often seek online help to troubleshoot problems of their home network that they are facing (Poole et al., 2009). Gathering the knowledge about millions of such end users spread all over the world researchers could easily produce a list that consists of the most commonly occuring home networking problems (Agarwal et al., 2009). Thus, the key to success is to both learn and share any new information with everybody else on the community as and when it appears. So, by collaborating the experiences from different such households the troubleshooting block rule engine can be made to adapt itself to such changes and consequently update its own rule engine. Given a considerable period of time the FHNS System would gradually evolve to an automated Expert System wherein it might suggest for example, a particular brand of network connected HDTV's creating some sort of a network configuration problem based upon the experience of other households. Thus, given an existing smart home network it can give a suggestion to the users before buying about the best possible alternatives of devices that are available in the market and which are compatible with their own home network thereby ensuring a quality and hassle-free service.

Ensuring Quality of Service (QOS) in terms of Internet Speed-Poor Internet speeds are a common cause of concern in almost every household. It can be due to a improperly configured network or due to policies set forward by the ISP itself. To detect situations wherein a user's ISP is the cause of performance degradation (relative to speed) Tariq et al. (2009) have developed the Network Access Neutrality Observatory (NANO) which collects network-flow statistics from different households and attempts to isolate the cause of such performance degradations based upon a statistical model. Thus, this opens up an opportunity to intermix the NANO agent with the FHNS System so as to improve its intelligence to understand the reasons of poor internet speeds if any and hence take appropiate measures.

As a means to improve Network Security Intrusion Detection Systems, antispywares, antivirus softwares and other network security algorithms depend heavily on their ability to collect different types of relevant data from as many sources as possible to keep themselves updated to the latest available threats (Hao et al., 2009; Perdisci et al., 2010; Ramachandran et al., 2007). Modern day scenario presents us with a very dangerous situation where the attackers could well be present in a smart home network. The problem is even more complicated because different home networks may be subscribed to different ISP's and generally they research in isolation to each other. Thus, a collaborative FHNS System should be in place wherein the FHNS Systems from different home networks interact among themselves, sharing the data they have with the sole aim of detecting any possible new vulnerabilities arising out of such network traffics.

\section{SPECIFIC REQUIREMENTS AND CHALLENGES FACED BY THE FHNS SYSTEM}

Issue of privacy and its legal implications: It is evident by this time that in order to ensure effective troubleshooting, FHNS systems from various homes should inter cooperate among themselves. In fact collecting, sharing and using the information about the events occurring in people's home networks is more challenging than the same prospects in the enterprise or service-provider 
networks (Allman and Paxson, 2007). But in doing so researchers risk sensitive user informations and their personal preferences like the type of websites visited, personal credit card informations and so on to be at stake. Obviously, no user would ever want any outsider to have a see into the daily happenings of their household which should be kept as a secret. But in doing so the very basic concept of collaborative information collection mechanism would be violated. To further complicate matters in a country like India the Information Technology Act poses a hefty penalty or imprisonment for upto a few years on the ISP's who violate the privacy of their customers.

Thus, the only solution that can be provided is to keep the FHNS System within the premises of a household only and to let the user of such a smart home network make a choice about which information is to be shared and which is to be not. Although, it might sound to be a conservative approach but right at this point of time it is the only best possible alternative available. Signing of a customer agreement form between the service provider and the customer may also be feasible solution. The concept of automatic operating system software updates will research well with the FHNS System too given their widespread acceptance. In that case, the FHNS System which is present in the household would regulary contact a centralized server of the service provider providing the FHNS service and keep the smart home network up to date.

Storage limitations: The problem of limited storage space is a very important one. The configuration that researchers used to test the system consisted of a modest $320 \mathrm{~GB}$ hard disk drive. Experiments revealed that for a full day of heavy Internet usage (consisting of 3 movie downloads, browsing the Internet and some e-mail exchanges) roughly $3 \mathrm{~GB}$ of disk space was utilized for storing the necessary records. This combined with the live streaming features being used on the HDTV's took up another 1 GB. Thus, the entire disc space would be consumed in no $>3$ months. Hence, periodic removal of the stored data to some offsite network storage device should be done at regular intervals. For example, data transfer from the FHNS system to any offsite network storage device can be scheduled at midnight of Sunday every week.

Reliability issue: The FHNS system researchers described should be robust and reliable. Specifically, it should be immune for an acceptable period of time to power failures or certain hardware configuration changes in the machine it is housed in. The design should be such that in the event of any hardware failure the loss of $\log$ data should be minimum.

\section{EXPERIMENTAL TEST BED AND SCOPE OF FUTURE WORK}

Researchers have implemented an initial prototype of the FHNS System as a tool to understand what exactly goes on in a home network. The prototype design is based upon an Intel based system running Windows 7 Home Premium Edition as the operating system. Further a software called CCProxy is also installed to handle multiple connections (both wired and wireless) simultaenously. Internet is accessed through high speed EVDO technology being provided by BSNL.

The FHNS System hardware has an Intel based system consisting of a Core $13-350 \mathrm{M}, 2.26 \mathrm{GHz}$ processor, 3 GB RAM, 500 GB hard disk drive, 2 Ethernet ports and support for Wireless LAN $(802.11 \mathrm{~b} / \mathrm{g} / \mathrm{n})$. Default configuration restricts the commencement of an Internet session from inside the house only. The FHNS features are primarily being provided by an open source packet monitoring tool called Wireshark that has been customized as per the requirement. Monitoring of packets by Wireshark is being done at the application, transport, network and data-link layer levels. A certain region in the hard disk drive has been reserved as the data storage and housekeeping block wherein all the packets that are being captured are stored. Certain rules have been developed that are applied to this section so that the stored packets are seggregated into the General-Purpose Subevent, Interesting Subevent and Unsusual Subevent block.

The algorithm that has been formulated to be the rule engine is fairly simple and has its base on the application layer and data-link layer only of the TCP/IP, Model. As the utility of the FHNS System depends primarily on the recorded data, researchers investigate the reliability of the Wireshark software to capture the packet events. Experiments were carried out on 3 different hosts in the home to simulate conditions of low, medium and heavy loading conditions. All the tests were carried out for a time span of $1 \mathrm{~h}$ and the percantage of packet loss was calculated. Light loading condition consisted of a music video download and general surfing of the websites. Medium loading condition consisted of 2 torrent downloads (Total file size $>=1 \mathrm{~GB}$ ) followed by the normal website surfing. Heavy loading condition consisted of 6 torrent downloads (file size $>=5 \mathrm{~GB}$ ), online video streaming using YouTube, video conferencing for $20 \mathrm{~min}$ 
Table 1: Below summarizes the results

\begin{tabular}{llll}
\hline Load type & Low & Medium & Heavy \\
\hline Time duration (h) & 1 & 1 & 1 \\
Packets captured & 46,265 & $1,36,999$ & $2,51,176$ \\
Percentage of packets lost & 0.032 & 0.101 & 0.332 \\
\hline
\end{tabular}

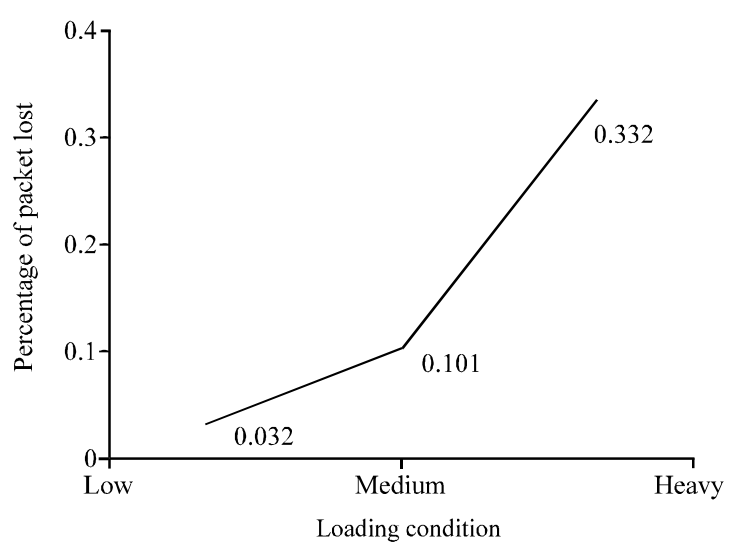

Fig. 3: A graphical plot of the loading condition on $\mathrm{X}$-axis $\mathrm{v} / \mathrm{s}$ the percentage of packets lost on the $\mathrm{Y}$-axis

using Skype apart from the normal website surfing. Table 1 shows the result of loading a graphical plot of the loading condition (i.e., packets captured) on the $\mathrm{X}$-axis $\mathrm{v} / \mathrm{s}$ the percentage of packets lost on the $\mathrm{Y}$-axis has been generated in Fig. 3 from the simulated results. The graph is of linear nature which gives us a clear indication that when using Wireshark as a packet monitoring tool the chances of packet loss increases proportionately as the network traffic increases. In fact towards, the heavy loading condition the curve becomes much more steeper indicating that the packet losses are even more in the higher end region.

\section{CONCLUSION}

It is evident from the experimental result that for the configuration that researchers use under heavy loading condition the percentage of packet loss becomes more. Thus, the FHNS System box that researchers use provides a satisfactory platform for the troubleshooting purpose. Some systems have been built that enables the home users to visualize the bandwidth been taken up by the different applications and hence control them in a proper manner (Chetty et al., 2010). Similarly the Eden System (Kandula et al., 2009) uses a customized router for data collection purposes and Home Network Data Recorder (HNDR) Systems (Calvert et al., 2010) tries to figure out the events that happened just prior to a problem occurrence. Similar approach has been attempted for Enterprise Networks also (Kandula et al., 2009).

\section{RECOMMENDATIONS}

In the near future, researchers expect to improve the capabilities of the prototype so that it can capture all the network events that have been described earlier. Researchers strongly have an intution that the techniques used by any Intrusion Detection System can be extended to the FHNS System also and so researchers intend to judiciously mix the functionalities of both. Researchers also have a vision to build up an Extensive Data Search Engine that will have intelligence of its own to detect the causes of home network disruption.

\section{REFERENCES}

Agarwal, B., R. Bhagwan, T. Das, S. Eswaran, V.N. Padmanabhan and G. Voelkar, 2009. Netprints: Diagonising home network misconfigurations using shared knowledge. Proceedings of the 6th USENIX NSDI, April 22-24, 2009, Boston, M.A., pp: 381-394.

Allman, M. and V. Paxson, 2007. Issues and etiquette concerning use of shared measurement data. Proceedings of the Internet Measurement Conference, October 23-26, 2007, San Diego, pp: 135-140.

Calvert, K., W. Edwards and R. Grinter, 2007. Moving towards the middle: The case against the end-to-end argument in home networking. Proceedings of the 6th Workshop on Hot Topics in Networks, November 14-15, 2007, Atlanta, CA., pp: 1-6.

Calvert, K.L., W.K. Edwards, N. Feamster and R.E. Grinter, Y. Deng and X. Zhou, 2010. Instrumenting home networks. Proceedings of the SIGCOMM Workshop on Home Networks, August 30-September 3, 2010, New York, USA., pp: 55-60.

Chetty, M., J.Y. Sung and R.E. Grinter, 2007. How smart homes learn: The evolution of the networked home and household. Proc. Ubicomp, Innsbruk, Austria, 4717: 127-144.

Chetty, M., R. Banks, R. Harper, T. Reagan and A. Sellen et al., 2010. Who's hogging the bandwidth: The consequences of revealing the invisible in the home. Proceedings of the Human Factors in Computing Systems(CHI) Conference, April 10-15, 2010 , New York, USA., pp: 659-668.

Grinter, R.E., W.K. Edwards, M.W. Newman and N. Ducheneaut, 2005. The work to make a Home Network Work. Proc. Eur. Conf. Comput. Supported Co-Operative Work, 18: 469-488.

Hao, S., N. Syed, N. Feamster, A. Gray and S. Krasser, 2009. Detecting spammers with snare: spatiotemporal network-level automatic reputation engine. Proceedings of the 18th USENIX Security Symposium, August 10-14, 2009, Montreal, Quebec, Canada, pp: 119-134. 
Kandula, S., R. Mahajan, P. Verkaik, S. Agarwal, J. Padhye and P. Bahl, 2009. Detailed diagnosis in enterprise networks. Proceedings of the SIGCOMM Conference on Data communication, October 2009, New York, USA., pp: 243-254.

Perdisci, R., W. Lee and N. Feamster, 2010. Behavioural clustering of Http-Based malware. Proceedings of the 7th USENIX NSDI, April 23-30, 2010, San Jose, CA., pp: 10 .

Poole, E.S., M. Chetty, T. Morgan, R.E. Grinter and W. Keith, 2009. Computer help at home: Methods and motivations for informal technical support. Proceedings of the 27th International Conference on Human Factors in Computing Systems, April 4-9, 2009, Boston, MA., USA., pp: 739-748.
Ramachandran, A., N. Feamster and S. Vempala, 2007. Filtering spam with behavioural blacklisting. Proceedings of the 14th Conference on Computer and Communications Security, October 28-31, 2007, Alexandria, VA., USA.

Sheehan, E., M. Chetty, R.E. Grinter and W.K. Edwards, 2008. More than meets the eye: Transforming the user experience of home network management. Proceedings of the Conference on Designing Interactive Systems, February 25-27, 2008, Cape Town, South Africa, pp: 487.

Tariq, M.B., M. Motiwala, N. Feamster and M. Ammar, 2009. Detecting network neutrality violations with casual inference. Proceedings of the 5th International Conference on Emerging Networking Experiments and Technologies, December 1-4, 2009, New York, USA. 